\title{
Effect of grain roughness on strength, volume changes, elastic and dissipated energies during quasi-static homogeneous triaxial compression using DEM
}

\author{
J. Kozicki · J. Tejchman · Z. Mróz
}

Received: 17 December 2011 / Published online: 5 May 2012

(C) The Author(s) 2012. This article is published with open access at Springerlink.com

\begin{abstract}
A quasi-static homogeneous drained triaxial compression test on cohesionless sand under constant lateral pressure was simulated using a three-dimensional DEM model. Grain roughness was modelled by means of symmetric clusters composed of rigid spheres imitating irregular particle shapes. The effect of grain roughness on shear strength, dilatancy, kinetic, elastic and dissipated energies was numerically analyzed. Some numerical results were compared with available experimental results.
\end{abstract}

Keywords Triaxial test · Granular material - Discrete element method · Grain roughness · Energy · Dissipation

\section{Introduction}

Granular materials consist of grains in contact and of surrounding voids, which change their arrangement depending on environmental factors and initial density. Their micromechanical and fabric behaviour is inherently discontinuous, heterogeneous and non-linear. To describe their behaviour, two main approaches are used: continuum and discrete ones. The first ones perform simulations at the global scale using the finite element method on the basis of e.g. elasto-plastic

\footnotetext{
J. Kozicki · J. Tejchman $(\bowtie)$

Faculty of Civil and Environmental Engineering,

Gdańsk University of Technology, Gdańsk, Poland

e-mail: tejchmk@pg.gda.pl

J. Kozicki

e-mail: jkozicki@pg.gda.pl

Z. Mróz

Polish Academy of Sciences, Institute of Fundamental Technological Research, Warsaw, Poland

e-mail: zmroz@ippt.gov.pl
}

and hypoplastic constitutive models enhanced by a characteristic length of micro-structure to describe strain localization (e.g. [7,13,38-41]). In turn, the latter ones perform simulations at the grain scale, i.e. each grain is modelled individually (e.g. $[2,4,16,24,30,31,42])$. Their advantages are that they directly model micro-structure and can be used to comprehensively study the mechanism of the initiation, growth and formation of shear zones at the micro-level which strongly affect macro-properties of granular matter. The disadvantages are: high computational cost, inability to model grain shape accurately and difficulty to validate it experimentally as the inertial and damping effects lose their meaning in quasi-static problems. However, they become more and more popular nowadays for modelling granular materials due to an increasing speed of computers and a connection possibility to the finite element method [33].

A large number of tests and simulations reveal that irregularly shaped grains strongly affect the quasi-static mechanical behaviour of granular materials $([26,34])$. To resemble the real grain shape (roughness), two main approaches are usually used: 1) contact moments between rigid spheres or disks are assumed $([5,16,18,21,28,44])$ or 2$)$ clusters of combined discrete elements that form irregularly-shaped grains are introduced [12,25, 26, 29, 35,48].

The concept of the total and stored elastic energy in soil mechanics was already discussed in the literature (see [8] and references therein). In the case of metals the elastic energy is divided into fully recoverable and stored or hidden portions. During unloading the recoverable part is released but the hidden energy remains stored in the material in the unloaded state as it represents the energy of residual microstresses at the grain and crystalline lattice levels. In soils and especially in granular materials the process of full unloading releases totally the grain interaction and related elastic energy. However, the partial unloading with the 
remained hydrostatic pressure can be considered and then the division into recoverable and stored elastic energies can be assumed. The free energy of a representative soil element can then be decomposed into elastic strain and irreversible (plastic) strain dependent portions $\Phi=\Phi^{e}\left(\varepsilon^{e}\right)+\Phi^{p}\left(\varepsilon^{p}\right)$ [9]. Here the first term represents the elastic strain dependent energy for a granular aggregate when only elastic grain deformation occurs with no sliding at contact interfaces and the second term represents the stored energy due to contact slip and sliding. It is not clear whether the concept of separation of energies is valid for granular materials since it is difficult to execute an elastic loading or unloading process for which the contact force interaction would occur only along normal directions to contact interfaces. In our study the evolution of the total elastic energy will be analyzed during the continuing deformation process without separation into recoverable and stored terms.

The objective of this paper is to present numerical analyses of quasi-static homogeneous true triaxial compression tests carried out to determine the macroscopic behaviour of sand specimens composed of discrete elements in the form of clusters. A three-dimensional discrete model YADE developed at University of Grenoble was used [21,22]. The particle breakage was not considered. The discrete simulation results were compared with the corresponding experimental data from drained axisymmetric triaxial compression tests performed by [45] at Karlsruhe University with real sand. The intention of our studies was to calculate the effect of the grain roughness (shape) on the shear strength, dilatancy, elastic and dissipated energies of real sand (so-called Karlsruhe sand), which had the same initial void ratio, mean grain diameter and grain distribution. A special attention was paid to the energy transformation in sand and its elastic and dissipative characteristics, playing a fundamental role in the granular matter behaviour $[1,2,37,46]$. The energy and dissipation results were compared with the similar ones from simplified two-dimensional simulations of biaxial compression with round particles performed by [23] and by [6]. In addition, the original own method was introduced to generate different grain shapes in the form of clusters of spheres.

\section{Discrete model}

The discrete element method (DEM) is widely used to model a range of processes across many industries $[2,10,14,19$, $23,32,42,47]$. To simulate the behaviour of sand, a threedimensional spherical discrete model YADE was developed at University of Grenoble [21,22] by taking advantage of the so-called soft-particle approach (i.e. the model allows for particle deformation which is modelled as an overlap of particles). A dynamic behaviour of the discrete (a)

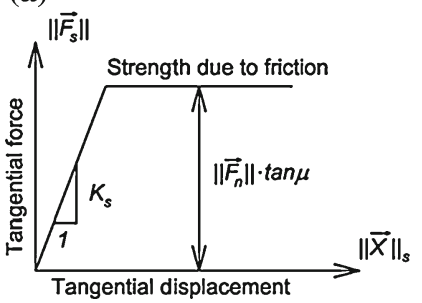

(b)

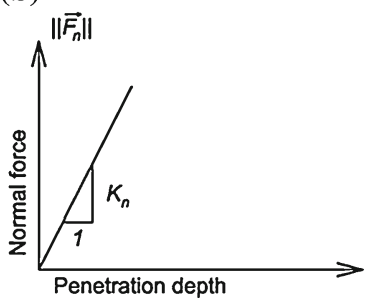

Fig. 1 Tangential and normal contact model $[21,36]$

system is solved numerically using a force-displacement Lagrangian approach and tracks the positions, velocities, and accelerations of each particle individually. It uses an explicit finite difference algorithm assuming that velocities and accelerations are constant in each time step. To calculate forces acting in particle-particle or particle-wall contacts, a particle interaction model is assumed in which the forces are typically subdivided into normal and tangential components. The total forces acting on each particle are summed. Next, the problem is reduced to the integration of Newton's equations of motion for both translational and rotational degrees of freedom. As the results, accelerations of each particle are obtained. The time step is incremented and accelerations are integrated over time to determine updated particle velocities and positions. To maintain the numerical stability of the method and to obtain a quick convergence to a quasi-static state of equilibrium of the assembly of particles, damping forces are introduced [10].

The interaction force vector $\vec{F}$ representing the action between two spherical discrete elements in contact is decomposed into a normal and tangential vector, respectively. A linear elasticity is chosen in our contact model. The normal and tangential forces are linked to the displacements through the normal stiffness $K_{n}$ and the tangential stiffness $K_{s}$ (Fig. 1a, b)

$\begin{aligned} \vec{F}_{n} & =K_{n} U \vec{N}, \\ \vec{F}_{s} & =\vec{F}_{s}+K_{s} \Delta \vec{X}_{s},\end{aligned}$

where $U$ is the penetration depth between elements, $\vec{N}$ denotes the normal vector at the contact point and $\Delta \vec{X}_{S}$ is the incremental tangential displacement. The tangential force $\vec{F}_{S}$ is obtained by summing its increments. The stiffness parameters are calculated with the aid of the modulus of elasticity of the grain contact $E_{c}$ and two neighbouring grain radii $R_{A}$ and $R_{B}$ (to determine the normal stiffness $K_{n}$ ) and with the aid of the modulus of elasticity $E_{c}$ and Poisson's ratio $v_{c}$ of the grain contact and two neighbouring grain radii $R_{A}$ and $R_{B}$ (to determine the tangential stiffness $K_{S}$ ), respectively [36]

$K_{n}=E_{c} \frac{2 R_{A} R_{B}}{R_{A}+R_{B}} \quad$ and $K_{s}=v_{c} E_{c} \frac{2 R_{A} R_{B}}{R_{A}+R_{B}}$. 
Fig. 2 Experimental results of axisymmetric triaxial tests by [45] with Karlsruhe sand (mean grain diameter $d_{50}=0.5 \mathrm{~mm}$ ): relationship between $\sigma_{1} / \sigma_{c}$ and $\varepsilon_{1}$, and between $\varepsilon_{v}$ and $\varepsilon_{1}$ at different confining pressures $\sigma_{c}$ : a initially very dense $\left(e_{o}=0.53\right)$ sand, b initially loose sand $\left(e_{o}=0.80\right)$ $\left(\sigma_{1}\right.$-vertical normal stress, $\varepsilon_{1}$-vertical normal strain, $\varepsilon_{v}$-volumetric strain) (a)

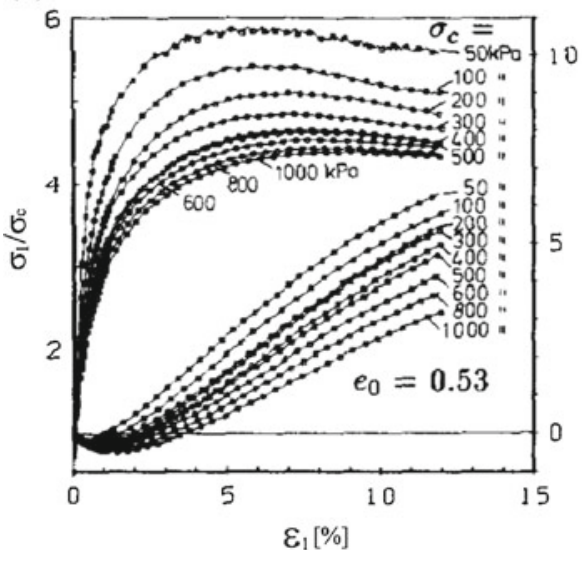

(b)

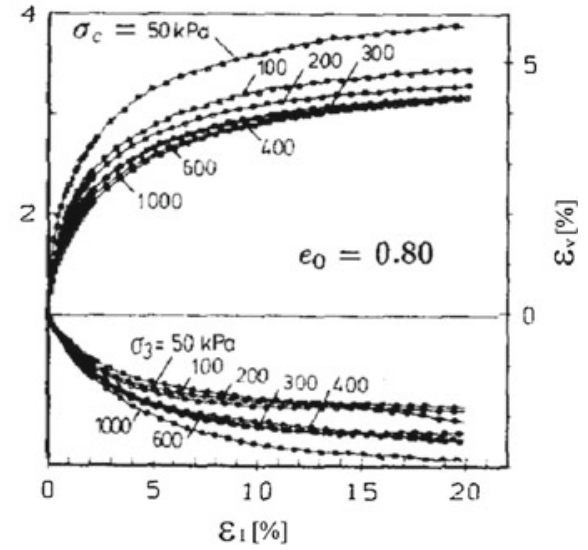

If the grain radius $R_{A}=R_{B}=R$, the stiffness parameters are equal to: $K_{n}=E_{c} R$ and $K_{s}=v_{c} E_{c} R$ (thus $K_{s} / K_{n}=$ $v_{c}$ ), respectively. The frictional sliding starts at the contact point if the contact forces $\vec{F}_{S}$ and $\vec{F}_{n}$ satisfy the frictional Mohr-Coulomb equation (Fig. 1a)

$$
\left\|\vec{F}_{S}\right\|-\left\|\vec{F}_{n}\right\| \tan \mu \leq 0
$$

with $\mu$ as the inter-particle friction angle (tension is not allowed). No forces are transmitted when grains are separated. The assumed tangential and normal contact relationships in the discrete model are demonstrated in Fig. 1a, b, respectively (the unloading is purely elastic). The elastic parameters $K_{n}$ and $K_{s}$ play a major role only in the elastic response of granulates. The normal stiffness modulus $K_{n}$ is related to the average modulus of elasticity of granular material $E$ and the Poisson ratio $v$. The ratio $K_{s} / K_{n}=v_{c}$ depends on the Poisson's ratio $v$ (the relationship is approximately linear, i.e. $E R \approx K_{n} / 5, v \approx v_{c} / 2$, cf. [5]). A choice of a linear elastic normal contact (Fig. 1b) is not in agreement with a non-linear Hertz interaction law between two adjacent elastic spheres with $F_{n} \sim U^{3 / 2}$ which is the exact elastic solution $[15,27]$. Therefore the elastic constants of the grain contact in our model with irregularly-shaped grains do not correspond to the elastic constants of the spheres' material in the Hertz law (thus $K_{n}$ in Eq. 3 is several times larger than the mean normal stiffness of the spherical grain material). The elastic contact moduli are specified from the experimental data of a triaxial compression sand test, as described later in the text.

To dissipate the excessive kinetic energy in the discrete system, a simple local non-viscous damping scheme was adopted, proposed by [11], which assumes a decrease of forces which increase particle velocities by using the damping parameter $\alpha$
$\vec{F}_{\text {damped }}^{k}=\vec{F}^{k}-\alpha \cdot \operatorname{sgn}\left(\vec{v}^{k}\right)\left|\vec{F}^{k}\right|$,

where $\vec{F}^{k}$ is the $k$ th component of the residual force vector and $\vec{v}^{k}$ is the $k$ th component of the translational velocity [36]. A positive damping coefficient $\alpha$ is smaller than 1 (sgn (•) returns the sign of the $k$ th component of velocity). The equations are separately applied to each $k$-th component of a $3 \mathrm{D}$ vector $x, y$ and $z$. Note that the effect of damping is insignificant in quasi-static calculations.

The following 3 main local material parameters are needed for discrete simulations: $E_{c}, v_{c}$ and $\mu$. In addition, the particle radius $R$, particle density $\rho$ and damping parameter $\alpha$ are required. The material parameters can be calibrated with corresponding axisymmetric triaxial laboratory test results on Karlsruhe sand by [20] and [45], Fig. 2. The index properties of Karlsruhe quartz sand are: mean grain diameter $d_{50}=$ $0.50 \mathrm{~mm}$, grain size among $0.08-1.8 \mathrm{~mm}$, uniformity coefficient $U=2$, maximum specific weight $\gamma_{d}^{\max }=17.4 \mathrm{kN} / \mathrm{m}^{3}$, minimum void ratio $e_{\min }=0.53$, minimum specific weight $\gamma_{d}^{\min }=14.6 \mathrm{kN} / \mathrm{m}^{3}$ and maximum void ratio $e_{\max }=0.84$. The sand grains are classified as sub-rounded/sub-angular.

In numerical simulations, a cubical sand specimen of $10 \times$ $10 \times 10 \mathrm{~cm}^{3}$ was used. A simplified linear grain distribution curve was used for Karlsruhe sand (grain range among 2.5$7.5 \mathrm{~mm}$, Fig. 3). To save the computation time, discrete simulations showing the capabilities of DEM were carried out with $d_{50}=5 \mathrm{~mm}$ (Fig. 3) instead of $d_{50}=0.5 \mathrm{~mm}$. The test was modelled using confining smooth rigid wall elements (without inducing shear localization). The top and bottom boundaries moved vertically as loading platens under straincontrolled conditions to simulate the confining pressure $p$. The loading speed was slow enough $(10 \mathrm{~mm} / \mathrm{s})$ to ensure the test was conducted under quasi-static conditions.

Different methods can be used to model irregularlyshaped grains $[3,12,17,25,26]$. In our method, each granular assembly was prepared by putting clusters of spheres 


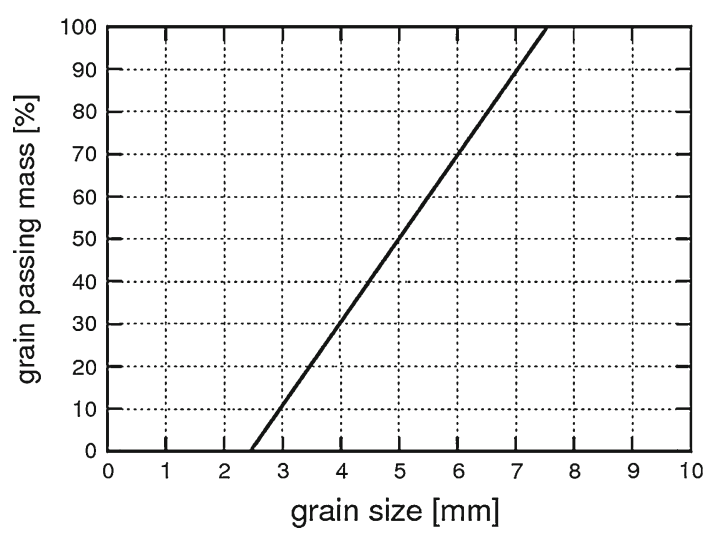

Fig. 3 Simplified linear grain distribution curve $\left(d_{50}=5.0 \mathrm{~mm}\right)$ used in discrete simulations to simulate Karlsruhe sand

of a random radius according to the grain distribution curve by Fig. 3 (without gravity) into a cubical container with 6 external walls which had a regular cubical grid with a particle distance of $10 \mathrm{~mm}$. The initial configuration of the specimen was thus isotropic. In order to obtain a desired initial density owing to grain overlapping, the inter-particle friction coefficient $\mu$ was varied between 0 and $30^{\circ}$ to exactly reproduce the target initial sand volumetric weight. During dynamic compression to the desired confining pressure $\sigma_{c}$, grains bounced against each other and moved in random directions, thus their initial ordered arrangement became random. The assembly was then allowed to settle to a state where the kinetic energy was negligible and the friction coefficient was set to $\mu=30^{\circ}$. The isotropic assembly was then subjected to the boundary driven triaxial compression. In general, arbitrary symmetric and non-symmetric shapes can be obtained with the different aspect, angularity and convexity index.

Figure 4 presents 12 different symmetric clusters of spheres used in discrete calculations. To generate e.g. clusters of 2 spheres (shapes 'b-f' of Fig. 4), the distance between spheres was set to be between 0 and $-0.33 d$. In turn, the clusters of 4 and 6 spheres (shapes ' $\mathrm{g}$, h' of Fig. 4) were made from 4 or 6 symmetrically placed spheres being tangential to the central point of the cluster. The discs (shape 'l' of Fig. 4) were created from 12 clumps of the type 'i' rotated around the main disc axis. Some shape indexes for the grain clusters of Fig. 4 are given in Table 1. The aspect index was defined as the ratio between the maximum and minimum cluster diameter, the convexity index ' 1 ' as the ratio between the smallest sphere volume encompassing the cluster and the cluster volume, and the convexity index ' 2 ' as the ratio between the smallest convex volume encompassing the cluster and the cluster volume.

In the case of the cluster of 2 spheres without the overlap (shape 'f' of Fig. 4), 26,300 clusters were composed of 52,600 spheres. In turn, 28,250 clusters were used with 197,750 spheres to model simple ellipsoids (shape 'i' of Fig. 4) and
14,500 clusters were used with 594,500 spheres to model disks (shape 'l' of Fig. 4). The computation time was 1 day (spheres), 2 days (cluster of 2 spheres), 4 days (ellipsoids) and 10 days (discs) using PC $3 \mathrm{GHz}$.

\section{Discrete results of homogeneous triaxial compression test}

The following discrete material parameters were used in simulations: $E_{c}=300 \mathrm{MPa}, v_{c}=0.3, \mu=30^{\circ}, \rho=25.5 \mathrm{kN} / \mathrm{m}^{3}$, $\alpha=0.08$ and $d_{50}=5.0 \mathrm{~mm}$ to match experimental results for real sand of Fig. 2.

\subsection{Effect of grain roughness on strength and volume changes}

Figure 5 shows the calculated evolution of the vertical normal stress $\sigma_{1}$ and overall void ratio $e$ versus vertical normal strain $\varepsilon_{1}$ for different clusters of spheres of Fig. 4 during triaxial compression with initially dense sand $\left(e_{o}=0.53, d_{50}=\right.$ $5 \mathrm{~mm}$ ) under confining pressure of $\sigma_{c}=200 \mathrm{kPa}$.

Similarly as in the real experiment (Fig. 2), the initially dense specimens exhibits initially elasticity, hardening (connected first to contractancy and then dilatancy), reaches a peak at about of $\varepsilon_{1}=2-3.5 \%$, gradually softens and dilates reaching at large vertical strain of $25-30 \%$ the same value of the vertical normal stress with the specimen deforming at constant volume, i.e. a critical state is always reached. Thus, the particle shape is not essential for the global critical internal friction angle (except of the case with single spheres). The both mobilized strength and dilatancy increase in general with increasing grain roughness and rolling resistance combined with an increase of the sphere number. Thus, the irregularly shaped particles provide obviously higher internal friction angles and have less tendency to rotate than perfect circular particles. The global maximum mobilized internal friction angle increases from $\phi_{\max }=28^{\circ}$ (spheres) up to $\phi_{\max }=48.9^{\circ}$ (ellipsoids 'k' of Fig. 4), respectively (Fig. 6). In turn, the global residual internal friction angle increases from $\phi_{c r}=25^{\circ}$ (spheres 'a' of Fig. 4) up to $\phi_{c r}=32^{\circ}$ (disks ' 1 ' and ellipsoids 'j' of Fig. 4), respectively (Fig. 5). The material dilatancy (volume increase) is the smallest with spheres and the highest with clumps ' $\mathrm{f}-\mathrm{k}$ ' of Fig. 4, respectively. The global elastic modulus is similar independently of the grain roughness $(E=70 \mathrm{MPa}$ with $v=0.25)$. The granular system shows small fluctuations in the residual phase. In general, the increase of the maximum internal friction angle is not directly connected with increasing three shape indexes above 1 of Table 1. Thus, the determination of the key geometrical grain parameter controlling the mechanical response (strength and volume changes) requires further investigations. 
(a)

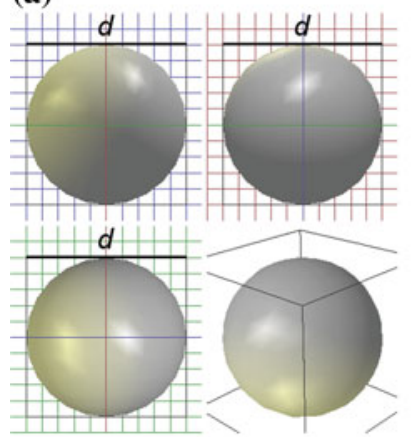

(d)

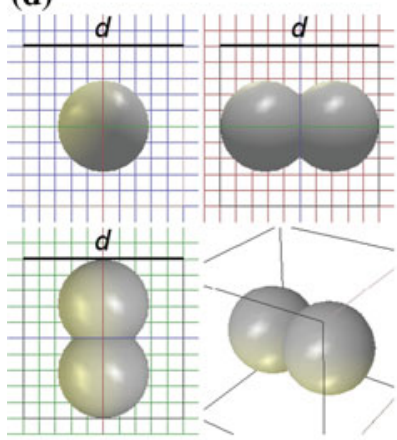

(g)

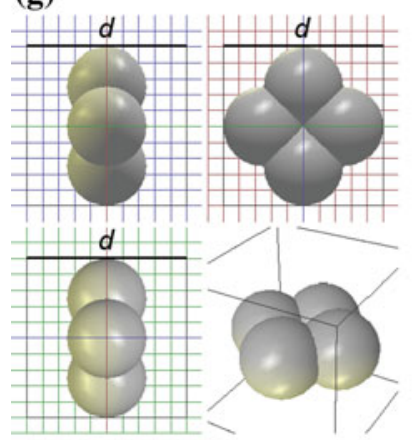

(j)

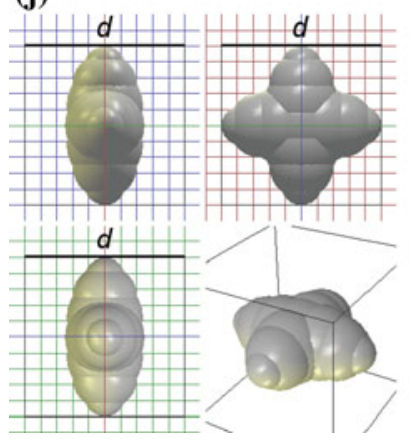

(b)

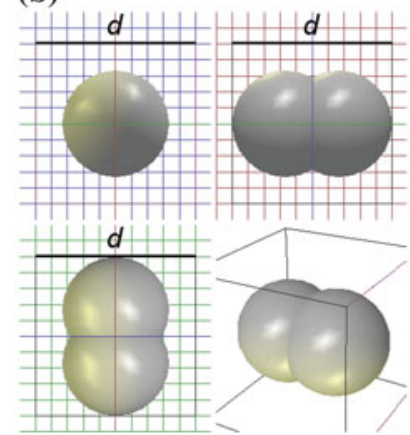

(e)

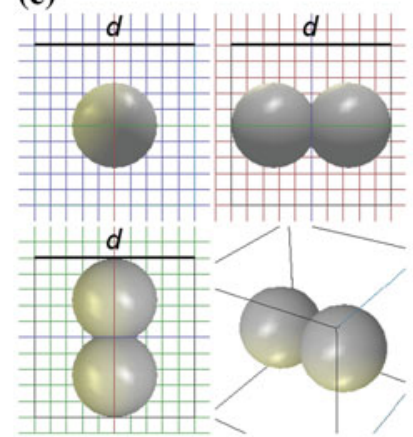

(h)

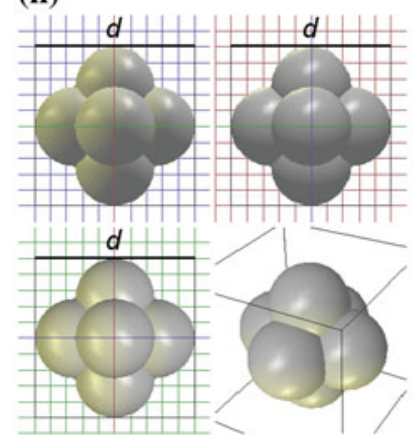

(k)

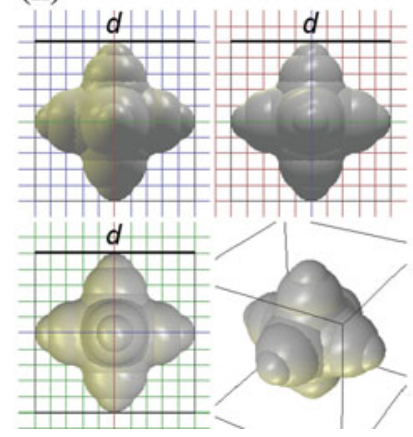

(c)

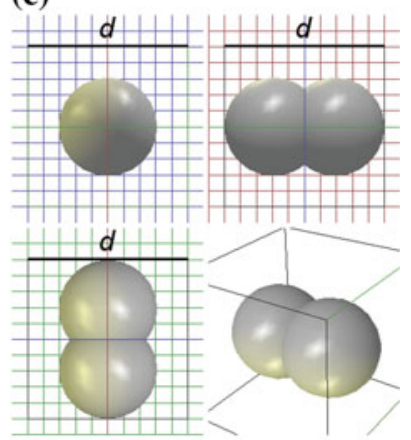

(f)

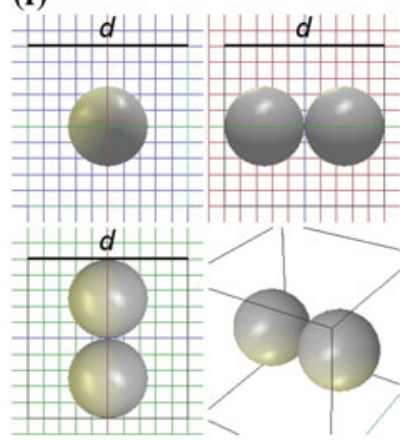

(i)

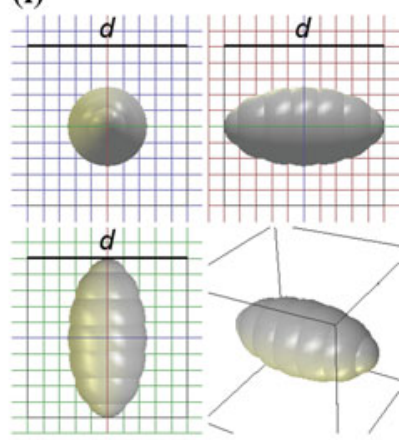

(I)

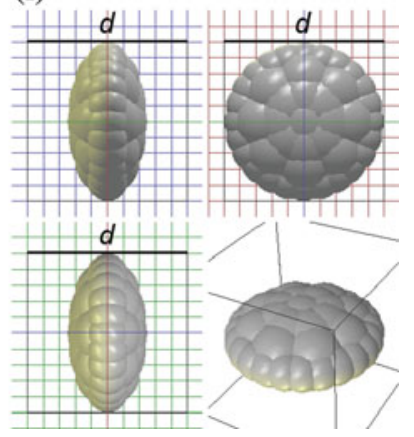

Fig. 4 Twelve different grain shapes (a-l) created by symmetric clusters of spheres used in discrete simulations $(d$-grain diameter)

Figure 6 shows a direct comparison between different granular clusters composed of 2 ellipsoids (shape ' $\mathrm{j}$ ' of Fig. 4), 2 spheres (shape 'd' of Fig. 4) and 6 spheres (shape 'h' of Fig. 4) and experimental axisymmetric triaxial compression results [45] on initially dense Karlsruhe sand $\left(e_{o}=0.53\right)$ at confining pressure of $\sigma_{c}=200 \mathrm{kPa}$.
The both experimental curves (global axial normal stress versus global axial strain and global volumetric strain versus global axial strain up to $\varepsilon_{1}=12 \%$ of Fig. 2) are satisfactorily reproduced. The calculated global maximum internal friction angle $\phi_{\max } \approx 43^{\circ}$ (calculated with principal stresses from the Mohr's equation) is slightly higher than the 
Table 1 Some shape indexes for grain clusters of Fig. 4

\begin{tabular}{llll}
\hline $\begin{array}{l}\text { Cluster shape of Fig. 4 } \\
\text { according to increasing } \\
\text { maximum internal friction }\end{array}$ & Aspect index & Convexity index '1' & Convexity index '2' \\
angle of Fig. 5 & & \\
\hline k) & 1.0 & 2.35 & 1.20 \\
g) & 2.0 & 2.26 & 1.15 \\
f) & 2.0 & 3.99 & 1.25 \\
h) & 1.0 & 1.67 & 1.14 \\
j) & 2.0 & 2.95 & 1.05 \\
e) & 1.875 & 3.32 & 1.17 \\
1) & 2.0 & 2.08 & 1.03 \\
d) & 1.75 & 2.81 & 1.11 \\
c) & 1.625 & 2.37 & 1.07 \\
i) & 2.0 & 4.15 & 1.04 \\
b) & 1.5 & 2.01 & 1.04 \\
a) sphere & 1.0 & 1.00 & 1.00 \\
\hline
\end{tabular}

(a)
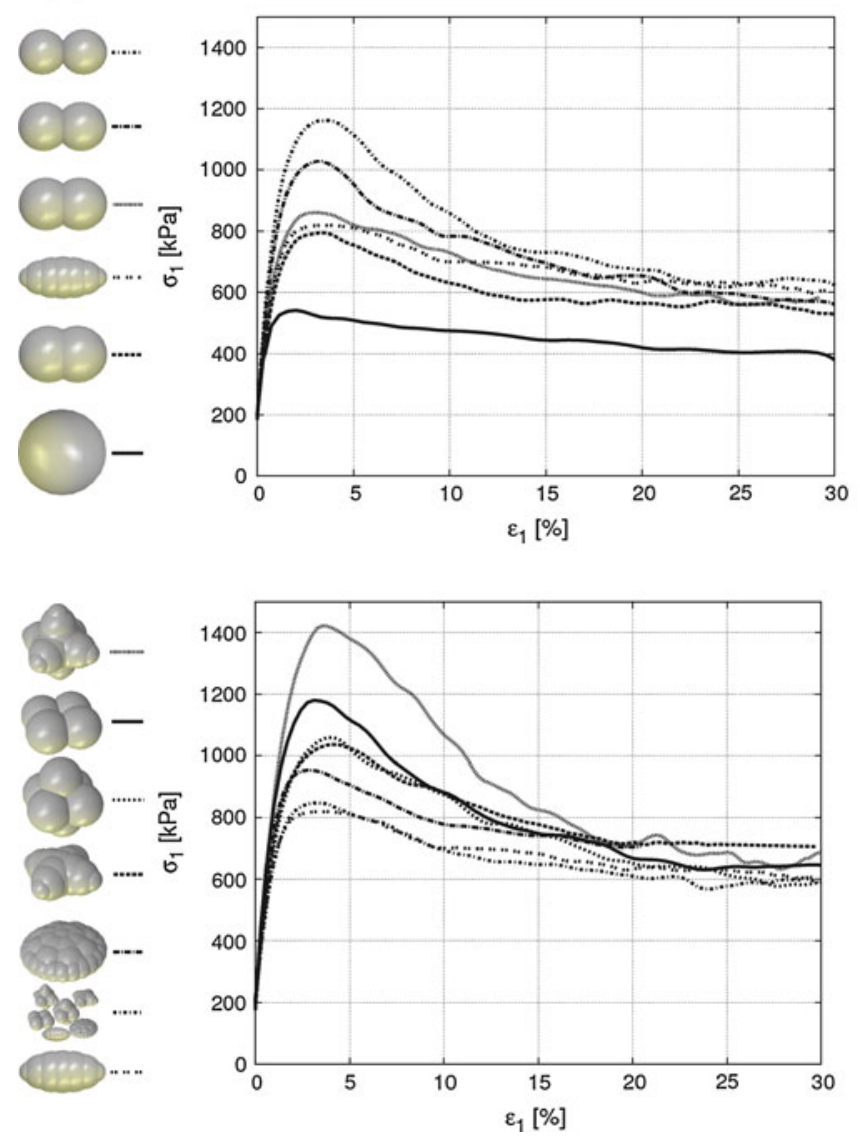

(b)
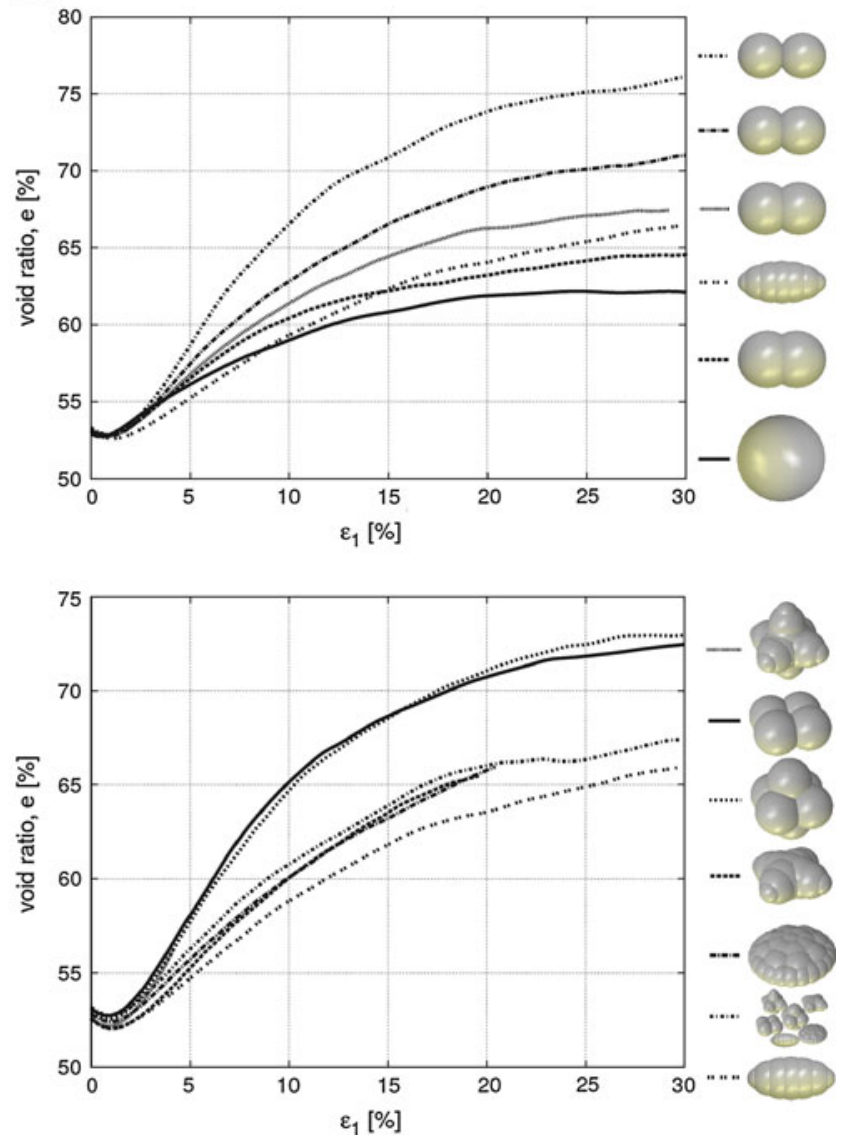

Fig. 5 Effect of particle roughness on vertical normal stress $\sigma_{1}$ versus vertical normal strain $\varepsilon_{1}(\mathbf{a})$ and void ratio $e$ versus vertical normal strain $\varepsilon_{1}$ (b) during homogeneous triaxial compression test for different grain shapes of Fig. $4\left(e_{o}=0.53, \sigma_{c}=200 \mathrm{kPa}, d_{50}=5.0 \mathrm{~mm}\right)$ 
Fig. 6 Effect of some clusters of spheres of Fig. 4 on vertical normal stress $\sigma_{1}$ versus vertical normal strain $\varepsilon_{1}$ (a) and volumetric strain $\varepsilon_{v}$ versus vertical normal strain $\varepsilon_{1}$ (b) from discrete simulations $\left(E_{c}=0.3 \mathrm{GPa}, v_{c}=0.3\right.$, $\mu=30^{\circ}$ ) compared to experiments of Fig. 2 during homogeneous triaxial compression test $\left(e_{o}=0.53, \sigma_{c}=\right.$ $200 \mathrm{kPa}, d_{50}=5.0 \mathrm{~mm}$ ): curve $a$ for grain shape ' $j$ ', curve $b$ for grain shape ' $h$ ', curve $c$ for grain shape ' $d$ ', curve $d$ for experiment
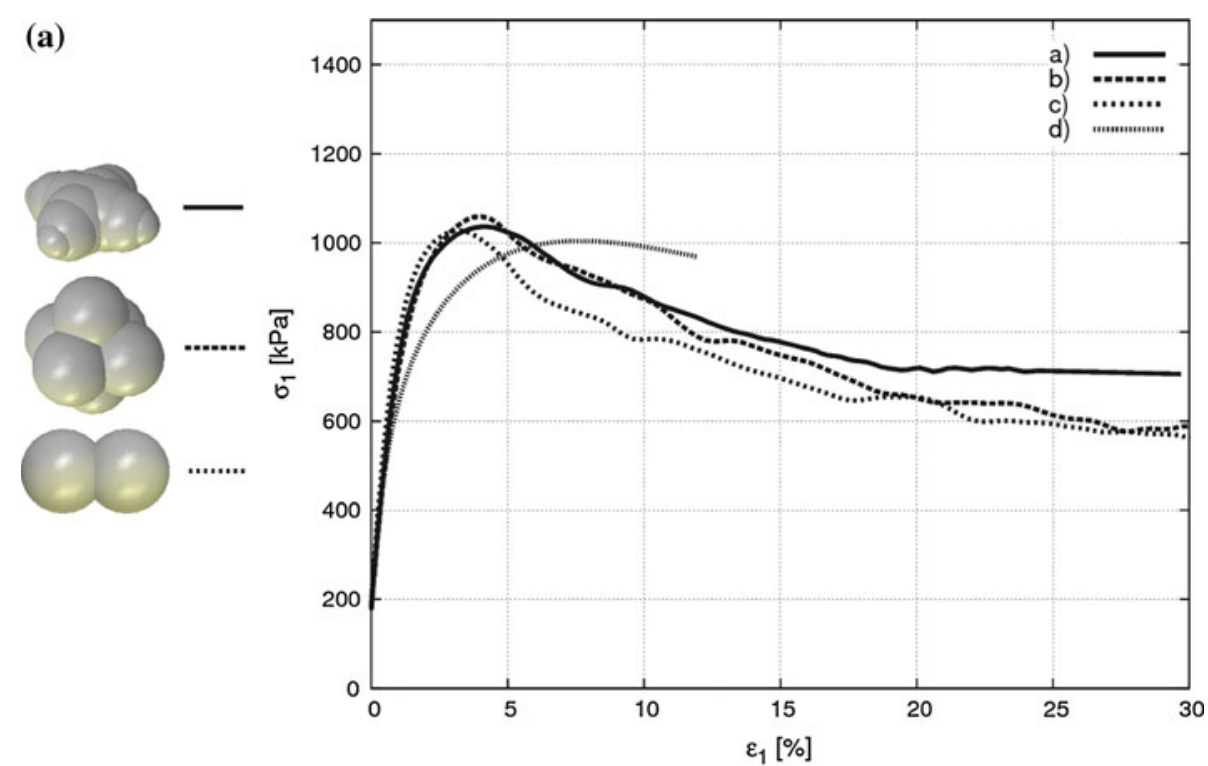

(b)
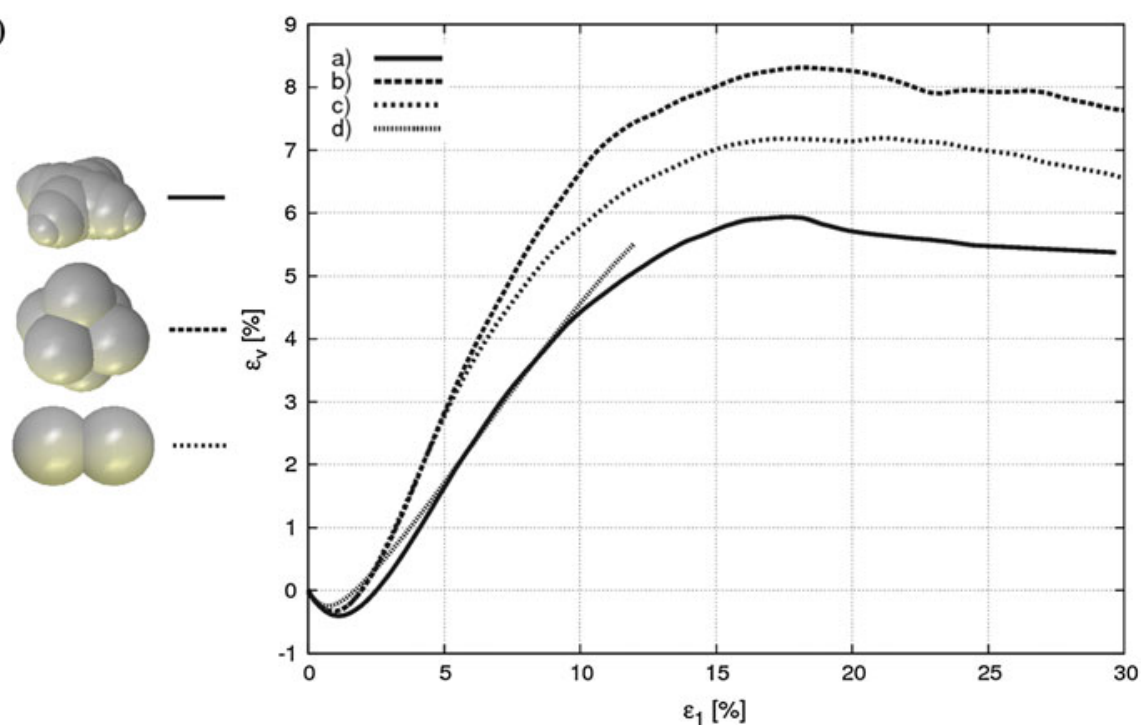

experimental value of $\phi=42^{\circ}$ (Fig. 2). The softening rate is similar. However, the calculated strain $\varepsilon_{1}=3 \%$ is about twice too small. It might be improved by decreasing the modulus of elasticity of the grain contact [5,43] or by introducing a non-linear contact model by Hertz-Mindlin-Deresiewicz (based on our preliminary calculations). The calculated dilatancy angles of $\psi=30^{\circ}-40^{\circ}$ match well the experimental outcome of $\psi=28.5^{\circ}$ (Fig. 2). The calculated global residual internal friction angle is $30^{\circ}-34^{\circ}$.

The numerical effect of initial void ratio $e_{o}$ and lateral pressure $\sigma_{c}$ on the sand behaviour using clusters of 6 spheres ' $h$ ' is described in Figs. 7 and 8. A decrease of initial void ratio causes an increase of the global maximum internal friction angle: $\phi_{\max }=31.0^{\circ}$ at $\varepsilon_{1}=20 \%\left(e_{o}=0.74\right), \phi_{\max }=$ $41.7^{\circ}$ at $\varepsilon_{1}=3 \%\left(e_{o}=0.54\right)$ and $\phi_{\max }=46.9^{\circ}$ at $\varepsilon_{1}=2.5 \%\left(e_{o}=0.46\right)$. The residual internal friction angle is always the same $\phi_{c r}=31.0^{\circ}$ independently of $e_{o}$. Initially dense sand undergoes initial contractancy and then dilatancy, and initially loose sand is subjected to contractancy only. The global maximum $\phi_{\max }$ and residual $\phi_{c r}$ internal friction angle slightly decrease with increasing lateral pressure for initially dense material: $\phi_{\max }=42.2^{\circ}, \phi_{c r}=29.1^{\circ}$, $\psi=47.5^{\circ}\left(\sigma_{c}=300 \mathrm{kPa}\right), \phi_{\max }=41.9^{\circ}, \phi_{c r}=30.6^{\circ}, \psi=$ $46.4^{\circ}\left(\sigma_{c}=200 \mathrm{kPa}\right)$ and $\phi_{\max }=40.3^{\circ}, \phi_{c r}=30.5^{\circ}, \psi=$ $32.0^{\circ}\left(\sigma_{c}=100 \mathrm{kPa}\right)$. The material dilatancy is similar.

\subsection{Effect of grain roughness on elastic and dissipated energies}

In a granular system there exist 3 main energies: elastic energy, kinetic energy and dissipated energy. In addition, the numerical dissipation also exists (see Eq. 5). The elastic 

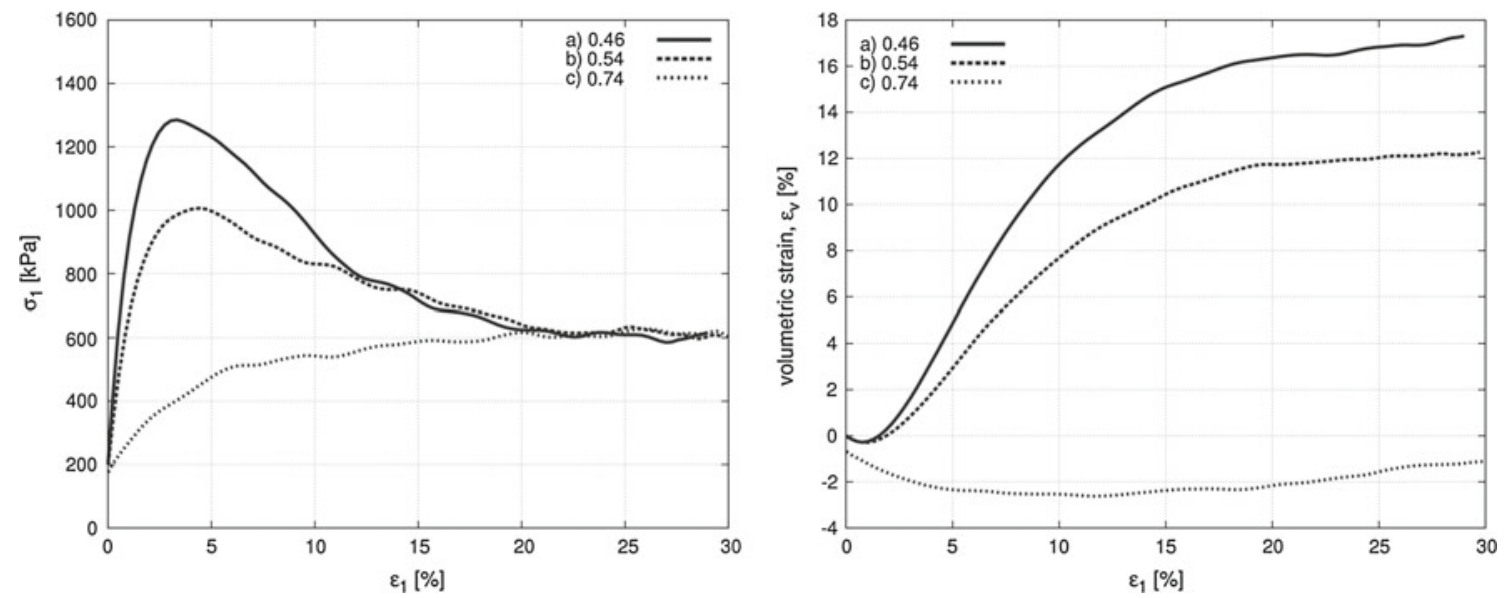

Fig. 7 Numerical effect of initial void ratio $e_{o}$ on stress-strain curve and volumetric strain $\varepsilon_{v}$ versus $\varepsilon_{1}$ during triaxial compression test using clusters composed of 6 spheres $\left(\sigma_{c}=200 \mathrm{kPa}\right): a e_{o}=0.46, b e_{o}=0.54, c e_{o}=0.74$
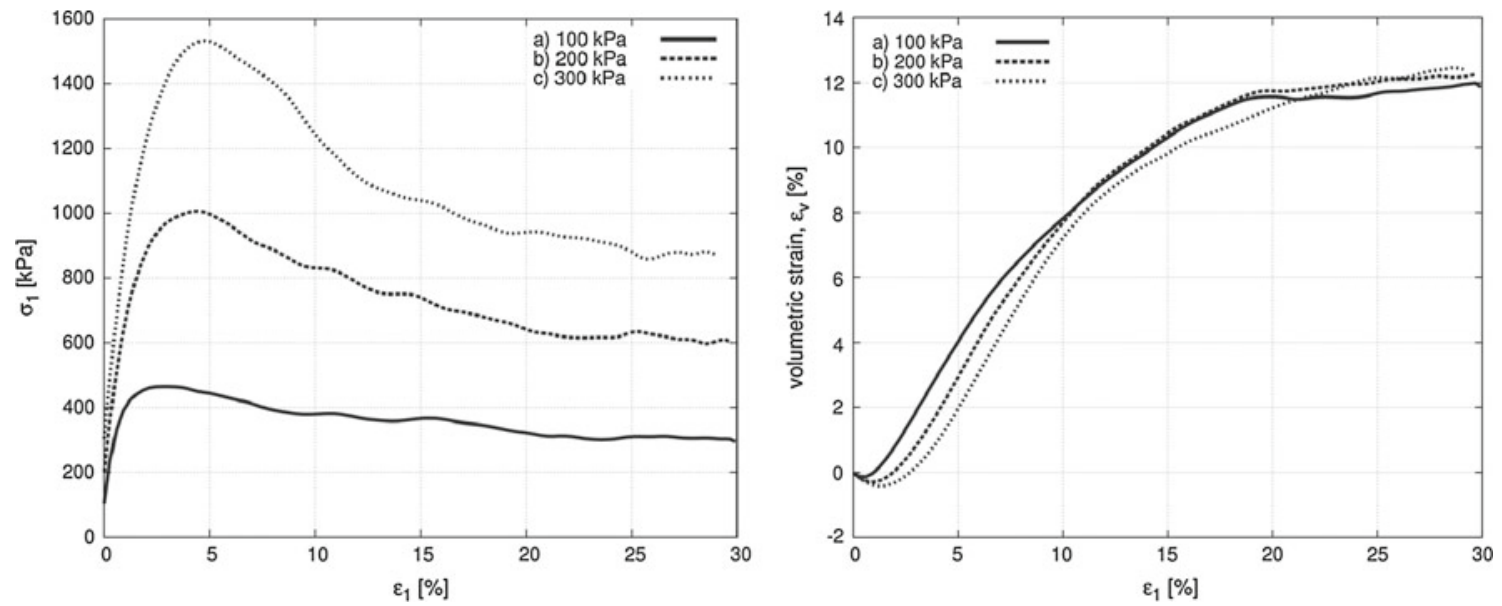

Fig. 8 Numerical effect of confining pressure $\sigma_{c}$ on stress-strain curve and volumetric strain $\varepsilon_{v}$ versus $\varepsilon_{1}$ during triaxial compression test using clusters composed of 6 spheres ' $h$ ' $\left(e_{o}=0.53\right)$ : $\boldsymbol{a} \sigma_{c}=100 \mathrm{kPa}, \boldsymbol{b} \sigma_{c}=200 \mathrm{kPa} \boldsymbol{c} \sigma_{c}=300 \mathrm{kPa}$

internal energy stored at contacts between grains $E_{e}$ is expressed in terms of work of elastic contact tangential forces $F_{S}$ on tangential elastic displacements $U_{t}$ and of contact normal forces $F_{n}$ on penetration depths $U$. In general, the elastic internal energy is expressed as follows (here $N$-the contact number)

$E_{e}=\sum_{1}^{N}\left(\frac{\left|F_{s}^{e}\right|^{2}}{2 K_{s}}+\frac{\left|F_{n}\right|^{2}}{2 K_{n}}\right)$

The kinetic energy $E_{c}$ of grains is caused by their translation and rotation

$E_{c}=\sum_{1}^{N}\left(\frac{1}{2} m v^{2}+\frac{1}{2} I \dot{\omega^{2}}\right)$

where $m$ is the mass and $I$ denotes the moment of inertia of a particle ( $v$-transitional velocity, $\dot{\omega}$-rotational velocity).
Due to quasi-static conditions, the effect of the kinetic energy $E_{c}$ is negligible (smaller than $1 \%$ of the elastic energy).

The dissipated energy $D_{p}$ is expressed in terms of work of tangential (shear) forces on related sliding displacements (see Fig. 1a)

$D_{p}=\sum_{1}^{N}\left(F_{s}^{p l} U_{s}^{\text {slip }}\right)$.

In addition, the numerical dissipation $D_{n}$ is specified during translation (see Eq. 5).

The total accumulated energy $E=E_{e}+E_{c}+D_{p}+D_{n}$ is equal to the external boundary work $W$ done on the assembly by 6 external forces on displacements of 6 rigid external walls

$W=\sum_{1}^{6} F \Delta u$. 

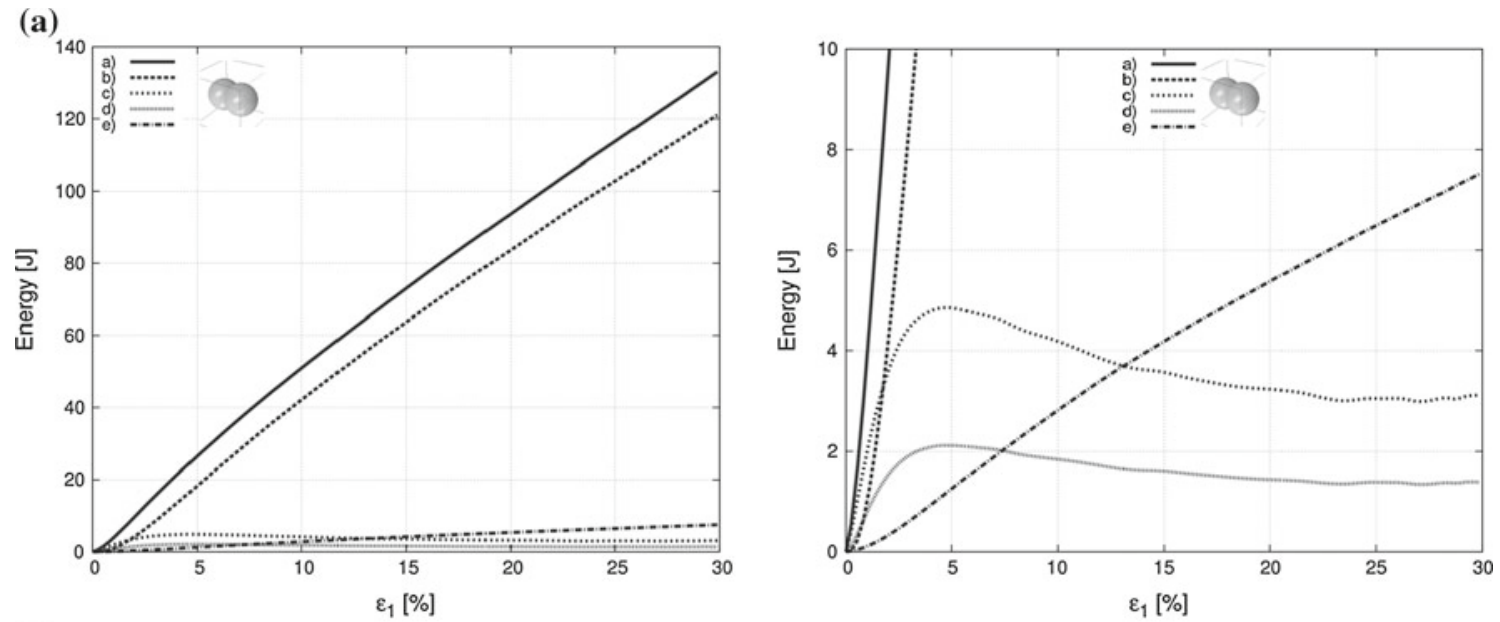

(b)

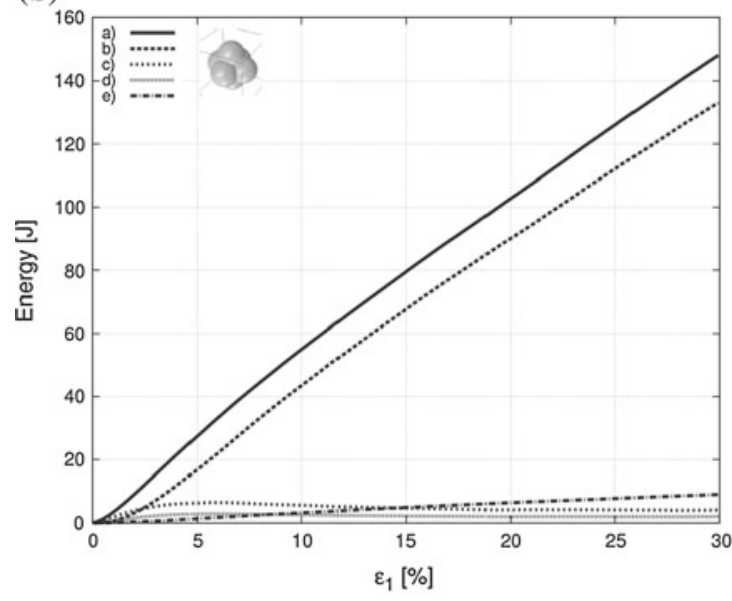

I

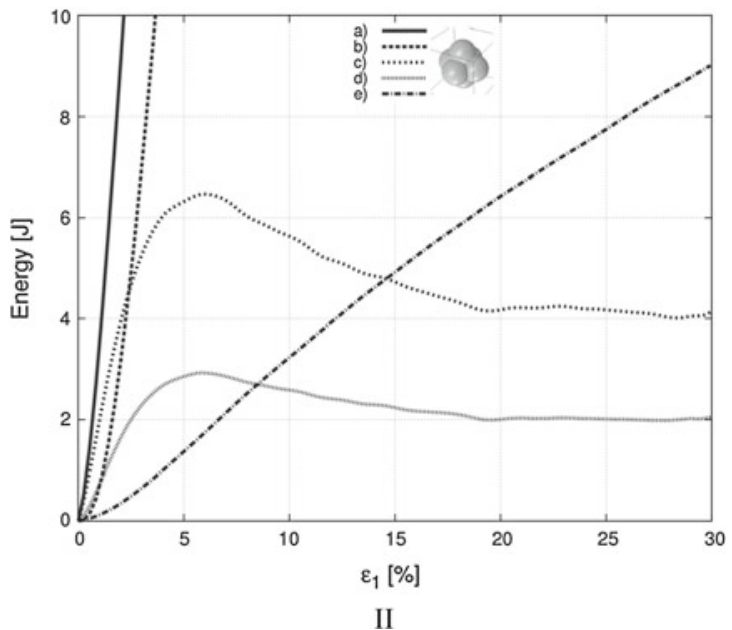

Fig. 9 Calculated evolution of: (a) total energy $E$, (b) plastic dissipation $D_{p},(c)$ elastic energy in normal direction $E_{c}^{n},(d)$ elastic energy in tangential direction $E_{c}^{s}$, (e) numerical non-viscous damping $D_{n}$ during

homogeneous triaxial compression test for: (a) clusters of 2 spheres, (b) clusters of 6 spheres $\left(e_{o}=0.53, \sigma_{c}=200 \mathrm{kPa}, d_{50}=5.0 \mathrm{~mm}\right)((\mathrm{I})$ wide view, (II) zoom)

(a)

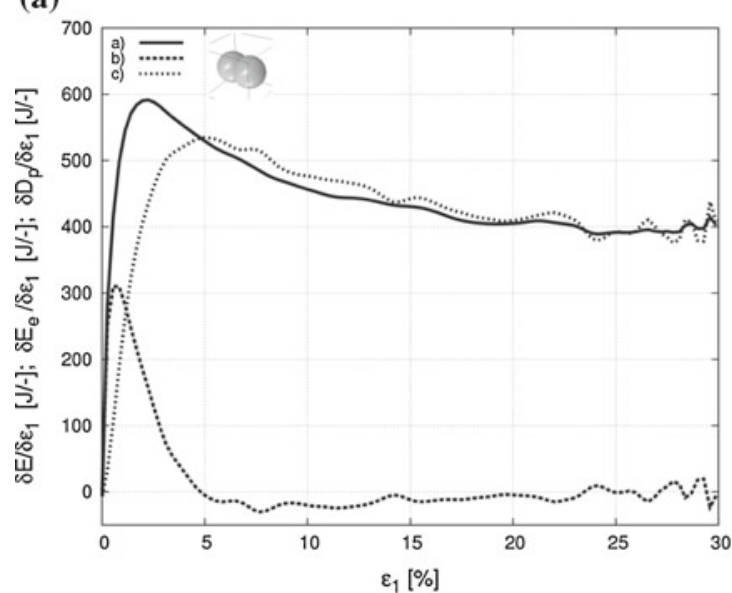

Fig. 10 Calculated evolution of: (a) external energy derivative $\delta E$ with respect to vertical normal strain $\varepsilon_{1}\left(\delta E / \delta \varepsilon_{1}\right),(b)$ elastic internal energy derivative $\delta E_{e} / \delta \varepsilon_{1}$ and $(c)$ plastic dissipation derivative $\delta D_{p} / \delta \varepsilon_{1}$ dur- (b)

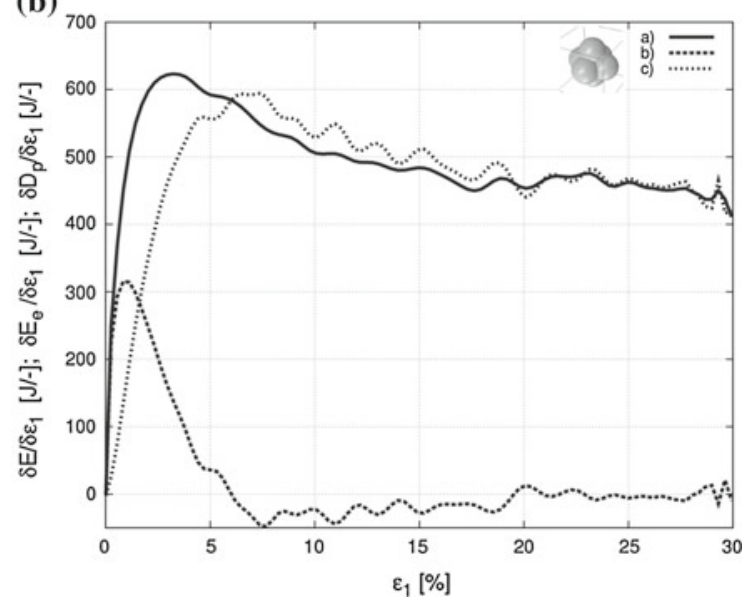

ing homogeneous triaxial compression test: (a) clusters of 2 spheres, (b) clusters of 6 spheres $\left(e_{o}=0.53, \sigma_{c}=200 \mathrm{kPa}, d_{50}=5.0 \mathrm{~mm}\right)$ 
(a)

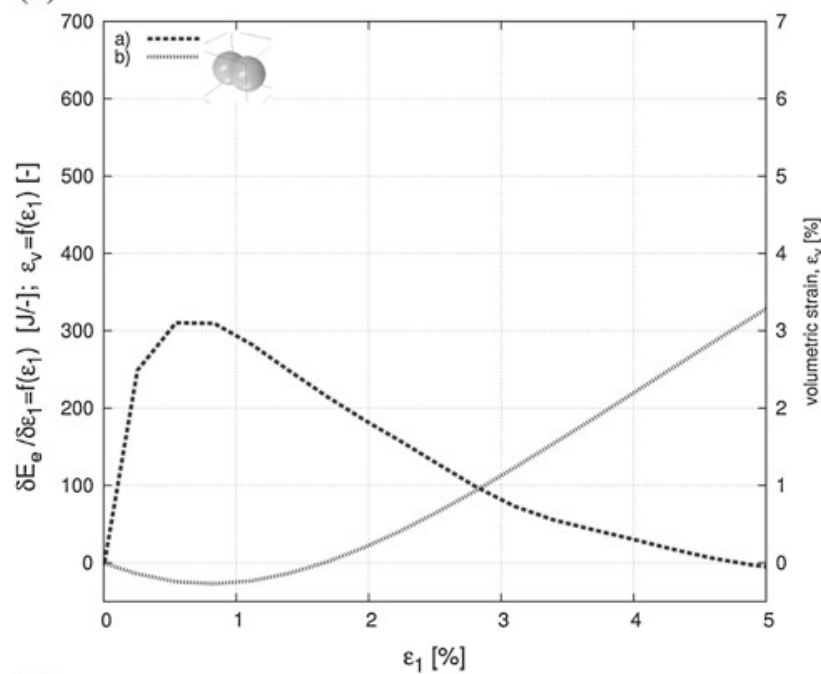

(b)

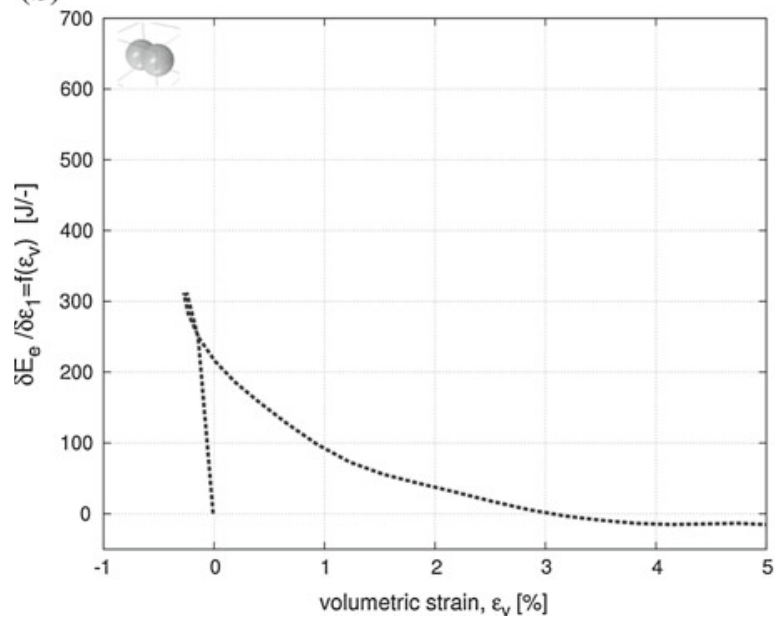

Fig. 11 Calculated initial evolution of elastic internal energy derivative $\delta E_{e}$ during homogeneous triaxial compression test (dotted line) with respect to vertical normal strain $\varepsilon_{1}$ as $\delta E_{e} / \delta \varepsilon_{1}=\mathrm{f}\left(\varepsilon_{1}\right)$ (curve $b$ describes evolution of volumetric strain $\left.\varepsilon_{v}=\mathrm{f}\left(\varepsilon_{1}\right)\right)$ (a) and with respect to vertical normal strain as $\delta E_{e} / \delta \varepsilon_{1}=\mathrm{f}\left(\varepsilon_{v}\right)$ (b) (clusters of 2 spheres, $\left.e_{o}=0.53, \sigma_{c}=200 \mathrm{kPa}, d_{50}=5.0 \mathrm{~mm}\right)$

Figure 9 shows the calculated effect of the grain roughness on the total accumulated energy $E$, elastic internally stored energy at contacts $E_{e}$, frictional dissipation $D_{p}$ and numerical damping $D_{n}$ in an initially dense sand $\left(e_{o}=0.53, p=\right.$ $200 \mathrm{kPa}, d_{50}=5.0 \mathrm{~mm}$ ). The systems of clusters of 2 spheres (shape 'd' of Figs. 4) and 6 spheres (shape 'h' of Fig. 4) were compared. In turn, the evolution of the external energy derivative $\delta E$, elastic internal energy derivative $\delta E_{e}$ and plastic dissipation derivative $\delta D_{p}$ with respect to the vertical normal strain $\varepsilon_{1}$ is demonstrated in Fig. 10. Figure 11 shows the initial evolution of the elastic internal energy derivative $\delta E_{e}$ with respect to the vertical normal strain $\varepsilon_{1}\left(\delta E_{e} / \delta \varepsilon_{1}\right)$ for clusters of 2 spheres. Finally, Fig. 12 demonstrates the evolution of the kinetic energy of granular systems. (a)

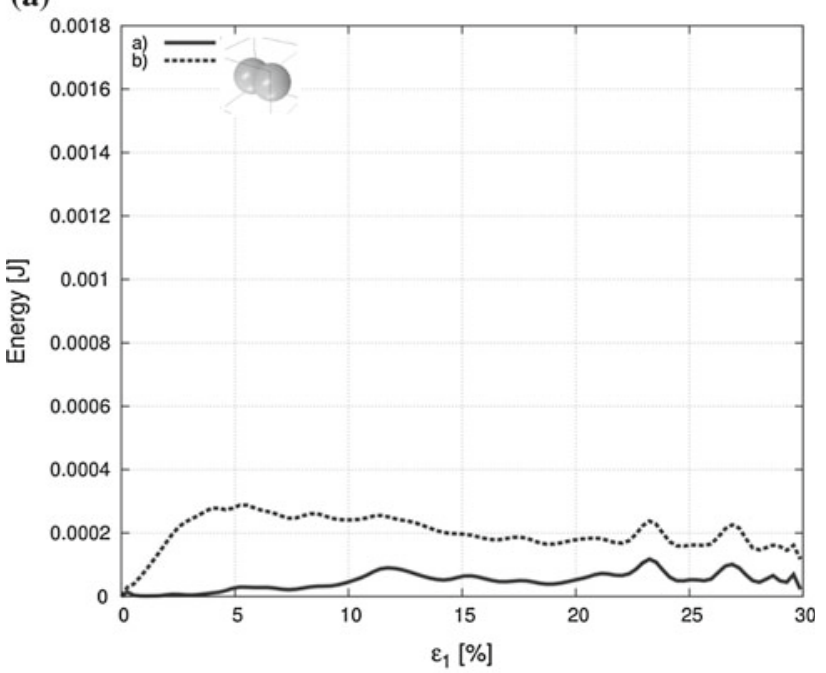

(b)

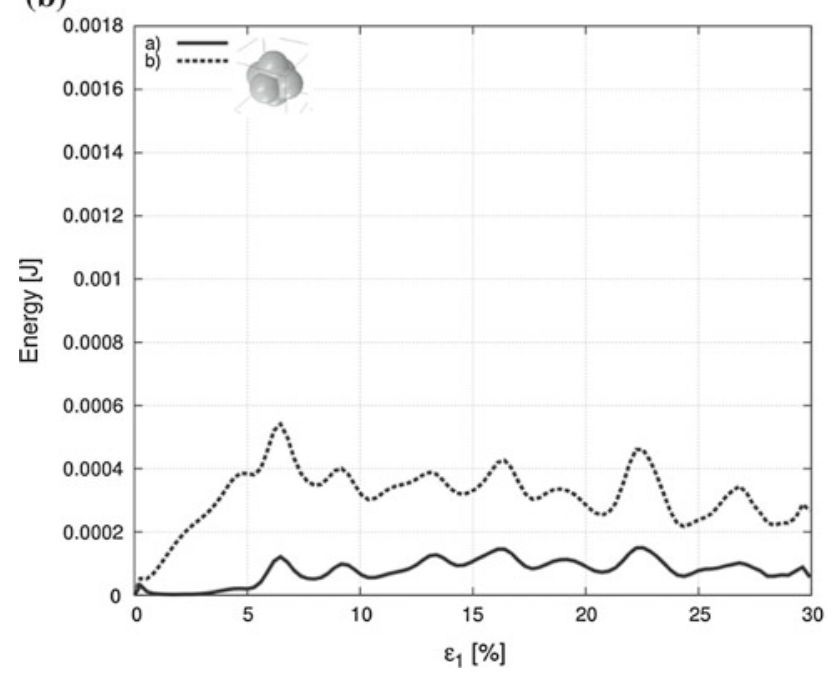

Fig. 12 Calculated evolution of kinetic energy $E_{c}$ during homogeneous triaxial compression test: (a) clusters of 2 spheres, (b) clusters of 6 spheres $\left(e_{o}=0.53, \sigma_{c}=200 \mathrm{kPa}, d_{50}=5.0 \mathrm{~mm}\right),(a)$ rotational kinetic energy, $(b)$ translational kinetic energy

There exists a roughly linear relationship between the total energy and plastic damping against the vertical normal strain (Fig. 9). The plastic dissipation during frictional sliding is equal to $50 \%$ of the total energy at $\varepsilon_{1}=3 \%$ (corresponding to the maximum vertical stress). At the residual state of $\varepsilon_{1}=30 \%$, it is already equal to $88 \%$ of the total energy. The numerical damping is equal always to $6 \%$ of the total energy. The evolution of the total elastic internal energy (and its components) is similar to the evolution of the shear strength; however the maximum value is at $\varepsilon_{1}=5 \%$ (Fig. 6). At the beginning of deformation at $\varepsilon_{1}<1 \%$ (when the specimen is in the elastic range), the total energy is almost fully converted into the elastic energy. The derivative $\delta E_{e}$ of the elastic internal work with respect to $\varepsilon_{1}\left(\delta E_{e} / \delta \varepsilon_{1}\right)$ is initially positive (Fig. 10$)$. It rapidly 
approaches zero, then becomes negative (since $E_{e}=f\left(\varepsilon_{1}\right)$ diminishes, Fig. 9II) at about $\varepsilon_{1}=5 \%$ (Fig. 10) and afterwards slightly increases approaching an asymptote at zero (since $E_{e}=f\left(\varepsilon_{1}\right)$ reaches a residual state, Fig. 9II). Beyond strains of $\varepsilon_{1}=5 \%$, almost the entire input work is dissipated due to plastic deformation and numerical damping (the external energy rate and dissipation rate are equal $\delta W \cong \delta D$ ). The energies and fluctuations of the energy rates increase with increasing grain roughness. The elastic energy decreases during a dilative deformation process only at $\varepsilon_{1}>1 \%$ (Fig. 11).

The elastic internal work is $80 \%$ at $\varepsilon_{1}=1 \%, 40 \%$ at $\varepsilon_{1}=3$ and $5 \%$ at $\varepsilon_{1}=30 \%$ of the total energy, respectively. The residual elastic internal work is performed by contact normal forces in $70 \%$ and contact tangential forces in $30 \%$. Thus, the largest internal work is performed by the contact normal forces (due to the lack of the plastic deformation). The elastic energy ratio is the same at the residual state.

The evolution curves in Fig. 9 are qualitatively similar to those demonstrated by [6]. In turn, the evolution curves in Fig. 10 are slightly different in the initial phase only than those shown by [23], who used periodic boundary conditions (instead of walls). The calculated energy quantities are different than in analyses by [6] using the software of PFC2D, wherein e.g. the calculated elastic energy was significantly higher: $90 \%$ (at $\varepsilon_{1}=3 \%$ ) and $20 \%$ (at $\varepsilon_{1}=5 \%$ ) of the total energy (probably due to the lack of the third dimension).

The kinetic energy is very small due to the quasistatic loading of the granular system (Fig. 12). A release of the elastic energy drives grains to move. At the elastic stage, the kinetic energy is close to zero. The translational kinetic energy increases up to $\varepsilon_{1}=5-7 \%$ and then slightly decreases. The rotational kinetic energy continuously increases (slightly). The kinetic energy show fluctuations at the residual phase ( 2 spheres) or already after the peak (6 spheres) which correspond to the evolution of the elastic energy and damping rate. The fluctuations increase with the grain roughness. The transitional kinetic energy is 2-5 times higher than the rotational one.

\section{Conclusions and future work}

The numerical simulations of a homogeneous triaxial compression test show that a discrete model is capable to reproduce the most important macroscopic properties of cohesionless granular materials without it being necessary to describe the granular structure perfectly. Comparing the numerical simulations with the experimental axisymmetric triaxial tests conducted for different initial void ratios and confining pressures shows that the discrete model is able to realistically predict the experimental results for real cohesionless sand.
The following detailed conclusions can be also drawn:

- The model is capable of closely reproducing the behaviour of cohesionless soils in the elastic, contraction and dilatancy phase and at the critical state. At large strains, the granular specimen reaches always a critical state independently of its initial density. The higher the confining pressure, the smaller are both the global friction and dilatancy.

- The sand grain roughness can be modelled by irregularlyshaped grains which may cause an increase of the strength and volume changes.

- At the elastic stage, the boundary external work is mainly converted into the elastic energy. At the residual state, the almost total external boundary work is dissipated by plastic deformation.

- The evolution of the elastic energy is inherently related to the dilation effect. In fact, the dilation reduces the normal contact forces and also the number of contact points. As the major contribution to the elastic energy is related to the normal forces, the elastic energy decreases during the dilative deformation process and tends to a steady state corresponding to a critical state condition.

- The kinetic energy shows fluctuations which correspond to the evolution of the elastic energy and damping rate. The fluctuations increase with the grain roughness and can appear already in the softening phase if the grains are rough enough. The transitional kinetic energy is higher than the rotational one.

Our research will be continued. The discrete simulations will be carried with sand during biaxial compression out by taking into account shear localization. The local phenomena occurring in a shear zone (such as buckling of granular columns, vortices, force chain cycles, periodic alternating dilatancy and contractancy) will be carefully studied.

Acknowledgments Scientific work has been carried out by the first two authors as a part of the Project: "Innovative resources and effective methods of safety improvement and durability of buildings and transport infrastructure in the sustainable development" financed by the European Union (POIG.01.01.02-10-106/09-01).

Open Access This article is distributed under the terms of the Creative Commons Attribution License which permits any use, distribution, and reproduction in any medium, provided the original author(s) and the source are credited.

\section{References}

1. Alonso-Marroquin, F., Vardoulakis, I., Herrmann, H., Weatherley, D., Mora, P.: Effect of rolling on dissipation in fault gouges. Phys. Rev. E 74, 031306 (2006) 
2. Alonso-Marroquin, F., Mühlhaus, H.B., Herrmann, H.: Micromechanical investigation of granular ratcheting using a discrete model of polygonal particles. Particuology 6, 390-403 (2008)

3. Alonso-Marroquin, F., Wang, Y.: An efficient algorithm for granular dynamics simulations with complex-shaped objects. Granul. Matter 11(5), 317-329 (2009)

4. Bardet, J.P.: Introduction to computational granular mechanics. In: Cambou, B. (ed.) Behaviour of Granular Materials, CISM 385, pp. 99-171. Udine, Springer (1998)

5. Belheine, N., Plassiard, J.P., Donze, F.V., Darve, F., Seridi, A.: Numerical simulations of drained triaxial test using $3 \mathrm{D}$ discrete element modeling. Comput. Geotech. 36(1-2), 320-331 (2009)

6. Bi, Z., Sun, Q., Jin, F., Zhang, M.: Numerical study on energy transformation in granular matter under biaxial compression. Granul. Matter 13, 503-510 (2011)

7. Brinkgreve, R.: Geomaterial models and numerical analysis of softening. Dissertation, Delft University, pp. 1-153. (1994)

8. Collins, I.F.: The concept of stored plastic work or frozen elastic energy in soil mechanics. Geotechnique 55, 373-382 (2005)

9. Collins, I.F., Houlsby, G.T.: Application of thermomechanical principles to the modelling of geotechnical materials. Proc. R. Soc. Lond. Ser. A 453, 1975-2001 (1997)

10. Cundall, P.A., Strack, O.D.L.: The distinct numerical model for granular assemblies. Geotechnique 29, 47-65 (1979)

11. Cundall, P.A., Hart, R.: Numerical modeling of discontinua. J. Eng. Comput. 9, 101-113 (1992)

12. Ferellec, J.F., McDowell, G.R.: A method to model realistic particle shape and inertia in DEM. Granul. Matter 12, 459-467 (2010)

13. Gudehus, G., Nübel, K.: Evolution of shear bands in sand. Geotechnique 54(3), 187-201 (2004)

14. Herrmann, H.J., Luding, S.: Modeling granular media on the computer. Continuum Mech. Thermodyn. 10(4), 189-231 (1998)

15. Hertz, H.: On the contact of elastic solids. J. Reine Angew. Math. 92, 156-171 (1882)

16. Iwashita, K., Oda, M.: Rolling resistance at contacts in simulation of shear band development by DEM. ASCE J. Eng. Mech. 124(3), 285-292 (1998)

17. Jerier, J.F., Richefeu, V., Imbault, D., Donze, F.V.: Packing spherical discrete elements for large scale simulations. Comput. Methods Appl. Mech. Eng. 199, 1668-1676 (2010)

18. Jiang, M.J., Yu, H.S., Harris, D.: A novel discrete model for granular material incorporating rolling resistance. Comput. Geotech. 32, 340-357 (2005)

19. Ketterhagen, W.R., Amende, M.T., Hancock, B.C.: Process modeling in the pharmaceutical industry using the discrete element method. Pharm. Res. Dev. 98, 442-470 (2009)

20. Kolymbas, D., Wu, W.: Recent results of triaxial tests with granular materials. Powder Technol. 60(2), 99-119 (1990)

21. Kozicki, J., Donze, F.V.: A new open-source software developed for numerical simulations using discrete modelling methods. Comput. Methods Appl. Mech. Eng. 197, 4429-4443 (2008)

22. Kozicki, J., Donze, F.V.: Yade-open DEM: an open-source software using a discrete element merhod to simulate granular material. Eng. Comput. 26(7), 786-805 (2009)

23. Kruyt, N.P., Rothenburg, L.: Shear strength, dilatancy, energy and dissipation in quasi-static deformation of granular materials. J. Stat. Mech. P07021, 1-10 (2006)

24. Luding, S.: Cohesive, frictional powders: contact models for tension. Granul. Matter. 10(4), 235-246 (2008)

25. Maeda, K., Sakai, H., Kondo, A., Yamaguchi, T., Fukuma, M., Nukudani, E.: Stress-chain based micromechanics of sand with grain shape effect. Granul. Matter 12, 499-505 (2010)

26. Matsushima, T., Chang, C.S.: Quantitative evolution of the effect of irregularly shaped particles in sheared granular assemblies. Granul. Matter 13, 269-276 (2011)
27. Mindlin, R.D., Deresiewicz, H.: Elastic spheres in contact under varying oblique forces. J. Appl. Mech. Trans. ASME 75, 327344 (1953)

28. Mohamed, A., Gutierrez, M.: Comprehensive study of the effects of rolling resistance on the stress-strain and strain localization behavior of granular materials. Granul. Matter 12(5), 527-541 (2010)

29. Ng, T.T.: Particle shape effect on macro- and micro-behaviors of monodisperse ellipsoids. Int. J. Numer. Anal. Methods Geomech. 33, 511-552 (2009)

30. Oda, M., Kazama, H.: Micro-structure of shear band and its relation to the mechanism of dilatancy and failure of granular soils. Geotechnique 48(4), 465-481 (1998)

31. Ord, A., Hibbs, B., Regenauer-Lieb, K.: Shear band emergence in granular materials - a numerical study. Int. J. Numer. Anal. Methods Geomech. 31, 373-393 (2007)

32. Pena, A.A., Lizcano, A., Alonso-Mattoquin, F., Herrmann, H.J.: Biaxial test simulations using a packing of polygonal particles. Int. J. Numer. Anal. Methods Geomech. 32, 143-160 (2008)

33. Rojek, J.: Discrete element modelling of rock cutting. Comput. Methods Mater. Sci. 7(2), 224-230 (2007)

34. Rothenburg, L., Bathurst, R.J.: Micromechanical features of granular materials with planar elliptical particles. Geotechnique 42, 79-7995 (1992)

35. Salot, C., Gotteland, P., Villard, P.: Influence of relative density on granular materials behaviour: DEM simulations of triaxial tests. Granul. Matter 11(4), 221-236 (2009)

36. Šmilauer, V., Chareyre, B.: Yade DEM Formulation. Manual (2011)

37. Tillemans, H.J., Herrmann, H.J.: Simulating deformations of granular solids under shear. Phys. A 217(3-4), 261-288 (1995)

38. Tejchman, J., Wu, W.: Numerical study on shear band patterning in a Cosserat continuum. Acta Mech. 99, 61-74 (1993)

39. Tejchman, J.: Influence of a characteristic length on shear zone formation in hypoplasticity with different enhancements. Comput. Geotech. 31(8), 595-611 (2004)

40. Tejchman, J.: FE modeling of shear localization in granular bodies with micro-polar hypoplasticity. In: Wu, W., Borja, R.I. (eds.) Springer Series in Geomechanics and Geoengineering, Springer, Berlin (2008)

41. Tejchman, J., Gorski, J.: Computations of size effects in granular bodies within micro-polar hypoplasticity during plane strain compression. Int. J. Solids Struct. 45(6), 1546-1569 (2008)

42. Thornton, C., Yin, K.K., Adams, M.J.: Numerical simulation of the impact fracture and fragmentation of agglomerates. J. Phys. D 29, 424-435 (1996)

43. Widulinski, L., Kozicki, J., Tejchman, J.: Numerical simulation of a triaxial test with sand using DEM. Arch. Hydro Eng. Environ. Mech. 56(3-4), 3-26 (2009)

44. Widulinski, L., Tejchman, J., Kozicki, J., Leśniewska, D.: Discrete simulations of shear zone patterning in sand in earth pressure problems of a retaining wall. Int. J. Solids Struct. 48(7-8), 1191-1209 (2011)

45. Wu, W.: Hypoplastizität als Mathematisches Modell zum Mechanischen Verhalten granularer Stoffe. Heft 129, Institute for Soiland Rock-Mechanics, University of Karlsruhe (1992)

46. Zhao, Z., Liu, C.S.: Energy dissipation and dispersion effects in granular media. Phys. Rev. E 78(031307), (2008)

47. Zhu, H.P., Zhou, Z.Y., Yang, R.Y., Yu, A.B.: Discrete particle simulation of particulate systems: theoretical developments. Chem. Eng. Sci. 62, 3378-3396 (2007)

48. Yan, Y., Ji, S.: Discrete element modelling of direct shear tests for a granular material. Int. J. Numer. Anal. Methods Geomech. 34, 978-990 (2010) 